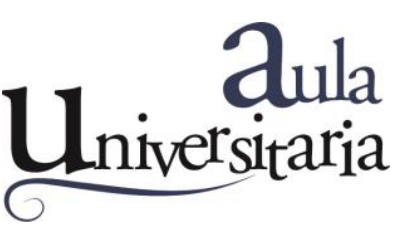

\title{
Enunciados coloquiales o simbólicos en la resolución de problemas de estadística aplicados a salud
}

\author{
WALZ, María F.; CONTINI, Liliana E. ${ }^{1}$; ÁVILA, Olga B. ${ }^{1}$
}

\section{Filiaciones institucionales}

${ }^{1}$ Docentes del Departamento de Matemática. Facultad de Bioquímica y Ciencias Biológicas. Universidad Nacional del Litoral.

\section{Correspondencia}

Facultad de Bioquímica y Ciencias Biológicas. Paraje El Pozo, s/n, 1er. Piso. Santa Fe de la Vera Cruz, Santa Fe, Argentina. Tel. 54-342-4575210

lecontini@gmail.com

\section{Resumen}

El lenguaje simbólico implica relacionar realidades y conceptos con un símbolo. Comprenderlo en el contexto de una ciencia supone realizar relaciones dialécticas entre el lenguaje de signos, las situaciones problema y el concepto científico que subyace detrás de cada símbolo. En la asignatura Estadística, de carreras orientadas a la salud, el empleo de signos, en general, tiene como objeto mostrar una intención para llevar a cabo una acción y no el de representar un objeto estadístico per sé.

El objetivo es evaluar si la resolución correcta de un ejercicio de distribución de probabilidad binomial se da en igual medida según las consignas estén enunciadas en forma simbólica o de manera coloquial.

Con tal fin, en los exámenes finales y parciales de Estadística de la Licenciatura en Nutrición de la Facultad de Bioquímica y Ciencias Biológicas se incorporó un problema de distribución binomial contextualizado con consignas expresadas simbólica y coloquialmente.

La resolución correcta del ejercicio se logró en mayor medida cuando el enunciado estaba escrito en símbolos y debía ser transcripto a un texto coloquial. Evidenciándose con esto que el lenguaje simbólico facilita una resolución mecanicista que puede prescindir de la comprensión significativa del tema. 


\section{Palabras clave}

expresiones simbólicas | expresiones coloquiales | distribución binomial | educación universitaria

\section{Summary}

Symbolic language implies relating realities and concepts with a symbol. Understanding it in the context of a science involves making dialectical relationships between sign language, problem situations and the scientific concept that underlies each symbol. In the subject Statistics of careers oriented to health, the use of signs, in general, aims to show an intention to carry out an action and not to represent a statistical object per se.

The objective is to evaluate if the correct resolution of a binomial probability distribution exercise is given in equal measure according to the slogans are stated in symbolic form or in a colloquial way.

To this end, in the final and partial exams of Statistics of the Licenciatura en Nutrición of the Facultad de Bioquímica y Ciencias Biológicas, a problem of binomial distribution contextualized with slogans expressed symbolically and colloquially was incorporated.

It was observed that the correct resolution of the exercise is not given in equal measure; resulting better when going from the symbolic to the textual / colloquial than the inverse; evidencing also that the symbolic language facilitates a mechanistic resolution that can dispense with the meaningful understanding of the subject.

\section{Keywords}

symbolic expressions / colloquiales expressions / binomial distribution / university education

\section{Introducción}

Leer es una construcción de sentido que conjuga procesos simultáneos que operan sobre información visual y no visual. La primera consiste en el reconocimiento de las diferentes unidades de lectura; y la segunda se basa en la construcción del sentido del texto (representaciones de alto nivel); cuyo éxito depende de lo que pueda aportar el lector desde sus conocimientos previos lingüísticos, simbólicos y culturales. Es decir, la cognición del lector provee información no visual; aporta el significado de las palabras o símbolos, los coteja con los conceptos y redes léxicas que posee e integra la información. El tiempo de esa integración está directamente relacionado con lo conocido de los signos que lee y la comprensión no es el resultado de la lectura sino de la significancia que haya logrado sobre el objeto aprendido y su condición de existencia (Alisedo, 1994).

Leer es descifrar la lengua escrita; la cual opera bajo la relación entre las unidades mínimas de escritura (grafemas) y las unidades mínimas de la lengua oral (fonemas). Entre ellas no existe ninguna relación natural. Por el contrario, fonemas y grafemas mantienen una relación arbitraria. Ninguna de estas dos unidades tiene significado aisladamente, pero sirven para distinguir significados. Segmentar, reconocer o analizar grafemas (sean estos letras o símbolos) y fonemas implica un proceso metalingüístico, en el que pueden darse dos vías: 
una subléxica o fonológica, que convierte grafemas en fonemas para luego acceder indirectamente al significado; y la otra, la léxica, directa, que es un reconocimiento inmediato del grafema que ha sido memorizado (Karmiloff Smith, 1994). En el caso de las representaciones lingüísticas de una ciencia particular en la que coexisten el objeto disciplinar mismo y el objeto lingüístico que lo expresa (D’Amore, et al. 2006), la comprensión de la notación simbólica, en general, va más allá de aprender a relacionar el símbolo con su objeto. Las relaciones dialécticas entre la idea, el lenguaje de signos y las situaciones problemas implican también la comprensión del concepto que subyace detrás de cada símbolo, en términos de hacer una abstracción de la realidad y explicarla a través de ellos. Así, el lenguaje simbólico, desde una perspectiva filosófica, consiste en relacionar realidades y conceptos con un símbolo, diferenciándose del lenguaje coloquial en el que, aunque se base en conceptos abstractos que necesitan ser entendidos, está naturalizado por el contexto en el que estamos inmersos desde el nacimiento y responde a un simple modelo de acciónreacción. Aunque en el primero los símbolos generalmente se utilizan como metodología de expresión abreviada de la oralidad de alguna disciplina, también pueden denotar un determinado concepto de la ciencia en cuestión. Por lo cual es importantísimo la cognición del signo en cuanto a lo que culturalmente codifica o se interpreta de este en cada institución o disciplina; dado que estos pueden sufrir transformaciones semióticas de un área a otra, que impidan el acceso al objeto representado o la conservación de su sentido (Duval, 2003) en cada una de ellas.

Según la lingüística sausseriana, los signos son una unión indisociable de dos elementos de naturaleza psíquica: el significado (concepto) y el significante (imagen asociada) y no simples marcas que representan cosas, lo cual sería una mera nomenclatura (Radford, 2006). Es decir, los signos significan algo cuando están inmersos en un sistema de signos relacionados con otros signos (aproximación estructuralista de significar los signos). En este sentido, Duval (1998) y Radford (2006) discuten acerca de las dificultades ocasionadas en el aprendizaje al no distinguirse lo funcional y lo estructural de un signo, cuando este tiene heterogeneidad semiótica.

En Estadística aplicada a ciencias de la salud, el uso de los símbolos, en general, tiene como objetivo abreviar una palabra o fonema lingüístico, mostrando con ellos una intención para llevar a cabo una acción, y no el de representar un objeto estadístico per sé; aunque encierren implícitamente un significado matemático que debe conocerse e interpretarse semánticamente en el contexto de la situación problema real en la que coexisten variabilidad en la literalidad de la semiótica.

En el ciclo básico del plan de carrera de la Licenciatura en Nutrición de la Facultad de Bioquímica y Ciencias Biológicas (FBCB) de la Universidad Nacional del Litoral (UNL) se inserta la asignatura Estadística. El porqué de su incorporación es debido al rol utilitario preponderante que ha adquirido esta ciencia en contextos de investigaciones científicas de todo tipo. En este sentido, el fin primordial de su inserción es que los futuros profesionales comprendan sus 
conceptos básicos para poder analizar e interpretar en términos de estos los resultados surgidos de estudios, y arribar a conclusiones confiables.

La simbología empleada en su enseñanza, tanto en los conceptos teóricos como en los ejercicios prácticos desarrollados en clases de esta asignatura, aplican más a concebir una traducción literal de lo simbólico a lo coloquial pero enmarcado en un problema aplicado del perfil profesional. Siendo esto, la cuestión que nos preocupa y ocupa; dado que hemos evidenciado ciertas dificultades para traducir el significado de un lenguaje a otro.

\section{Objetivo}

Evaluar la resolución de un ejercicio de distribución de probabilidad binomial según las consignas estén enunciadas en forma simbólica o de manera coloquial.

\section{Metodología}

Este estudio se llevó a cabo con alumnos de Estadística de la Licenciatura en Nutrición de la FBCB de la UNL. Asignatura correlativa con Matemática, correspondiente al segundo cuatrimestre de segundo año de la carrera. En el primer examen parcial programado en la planificación de la misma se planteó un ejercicio sobre el tema «distribución de probabilidad binomial» con cinco ítems. En los dos primeros se solicitó: estimar una probabilidad asociada a un evento de una variable aleatoria con distribución de probabilidad binomial (ítem a) y el valor esperado de la misma variable (ítem b), ambos bajo un lenguaje técnico/simbólico. El ítem «C» requería interpretar los resultados de los dos ítems anteriores, expresándolo en un texto en términos del problema. En el ítem «d», se les pedía calcular una probabilidad asociada a la ocurrencia de un rango de valores de la variable, pero estaba escrito en forma coloquial, por lo que debían interpretar la consigna contextualizada al problema, conocer la funcionalidad del símbolo de una desigualdad y traducir el texto a la simbología técnica requerida para poder resolver el ejercicio. En el ítem «e» se pedía nuevamente estimar el valor esperado de la variable, pero fue enunciado en un texto que expresaba el significado estadístico del concepto.

«Una dieta empleada en niños con bajo peso, diseñada especialmente para que estos alcancen el peso corporal óptimo, es efectiva en el $80 \%$ de los casos en los que se la aplica. En un centro de salud se tratan con tal dieta a cinco niños en situación de bajo peso y se define la variable «cantidad de niños que alcanzan el peso ideal por grupo de cinco niños tratados» $(X)$, estimar a) $P(X \leq 1)$ y b) estimar $\mathrm{E}(\mathrm{X})$; $\mathrm{c})$ Interpretar en forma coloquial en términos de la situación real, los resultados obtenidos en cada uno de los ítems anteriores; d) ¿Cuán probable es que como mucho uno de los cinco niños adquieran el peso saludable; e) Por 
cada grupo de diez niños con bajo peso tratados con esta dieta, en promedio, ¿cuántos alcanzan el peso saludable?

Para poder resolver los ítems «a» y «b» el alumno debe ir del grafema al fonema, reconociendo en la escritura simbólica el lenguaje textual abreviado en cada símbolo. También necesita conocer el significado del signo menor o igual y saber emplear la mecánica de cálculo involucrada en el tema; aunque no necesariamente debe haber aprendido significativamente los conceptos estadísticos involucrados. El ítem «c» implica reconocer en el resultado numérico el contenido de la proposición; lo cual puede hacerse sin necesidad de interpretar verdaderamente el significado probabilístico que encierra. Al respecto, se puede dar la respuesta correcta con solo traducir textualmente el significado abreviado de los símbolos enunciados en «a» y «b», con el agregado de la comprensión del concepto matemático implicado en la igualdad/desigualdad. El ítem «d» es igual al «a» pero está expresado en forma coloquial. En esta circunstancia, el alumno debe poder asociar la expresión «como mucho uno» con el símbolo de desigualdad «menor o igual a uno», interpretar lo que se le pide coloquialmente en términos de probabilidad, traducir correctamente el texto a la abreviatura simbólica empleada en la técnica de cálculo asumida en la institución y resolverlo. El último ítem, por su parte, requiere que el estudiante comprenda significativamente el concepto de valor esperado de una variable aleatoria y pueda disociar el significado (concepto) del significante (imagen asociada).

\section{Resultados}

El tamaño muestral fue 104. Los resultados obtenidos se describen en la siguiente tabla:

\begin{tabular}{|c|c|c|c|c|}
\hline Ítem & Bien & Regular & Mal & No resuelve \\
\hline a) & $71 \%$ & $1 \%$ * & $25 \%$ ** & $3 \%$ \\
\hline b) & $82 \%$ & $0 \%$ & $0 \%$ & $18 \%$ \\
\hline c) & $\begin{array}{l}\text { - Interpretación co- } \\
\text { rrecta del ítem a): } 45 \% \\
\text { - Interpretación co- } \\
\text { rrecta del ítem b): } 78 \%\end{array}$ & -- & $\begin{array}{l}\text { - Interpretación incorrecta } \\
\text { del ítem a): } 43 \%{ }^{* * *} \\
\text { - Interpretación incorrecta } \\
\text { del ítem b): } 0 \%\end{array}$ & $\begin{array}{c}\text { - Interpretación del } \\
\text { ítem a): } 12 \% \\
\text { - Interpretación del } \\
\text { ítem b): } 22 \%\end{array}$ \\
\hline d) & $53 \%$ & $1 \%$ * & $10 \%$ **** & $36 \%$ \\
\hline e) & $31 \%$ & $0 \%$ & $0 \%$ & $69 \%$ \\
\hline
\end{tabular}


Se considera como «Bien» resuelto un ítem, si este fue bien planteado y desarrollado y con resultado numérico correcto. Así mismo, para el caso de los ítems que requerían enunciar interpretaciones de resultados numéricos; estas fueron asumidas como correctas si las expresiones coloquiales eran lógicas para el concepto estadístico, tanto si eran frases libres o traducciones textuales de la simbología al texto. La consideración de resolución «Regular» se debió a que el ejercicio estuvo bien planteado, pero con errores de cálculo que no permitieron arribar al resultado correcto $\left(^{*}\right)$.

La categorización «Mal» resuelto, se debió a diferentes razones según el ítem. Esto es, por no considerar en el ítem «a» la probabilidad acumulada que implica el símbolo menor o igual $\left({ }^{* *}\right)$. En el ítem «C» se debió en todos los casos a la mala interpretación coloquial del resultado del cálculo correspondiente a lo solicitado como $\mathrm{P}(\mathrm{X} \leq 1)$, en términos de no asociar el significado del símbolo menor o igual con expresiones tales como «como mucho», «a lo sumo», «como máximo» $\left({ }^{* * *}\right)$.

Por último, la «Mal» resolución del ítem d) se debió en un $67 \%$ al hecho de no asociar la expresión coloquial «como mucho uno» con la probabilidad acumulada relacionada al símbolo menor o igual y en un 33\% por interpretar en el sentido inverso el símbolo (mayor o igual). Es decir, en lugar de plantear la $P(X \leq 1)$ plantearon $P(X \geq 1)\left({ }^{* * * *}\right)$.

\section{Discusión}

Los ítems enunciados de manera simbólica (ítems «a» y «b») son resueltos correctamente por una alta proporción de alumnos; mientras que, en contraposición, no se da en igual medida un buen desarrollo de un ejercicio contextualizado y enunciado coloquialmente (ítem «d»).

Por su parte, la interpretación de resultados numéricos fue realizada por la gran mayoría mediante una traducción en orden sistemático literal de los símbolos al texto, considerando que estos representan la abreviatura de cada palabra castellana. Esto lleva a pensar que hay, más bien, una dinámica mecanicista de resolución de los problemas que significativa conceptual.

En cuanto al hecho de haber un elevado porcentaje de alumnos (69 \%) que obtuvieron correctamente la $\mathrm{P}(\mathrm{X} \leq 1)$ y el valor esperado cuando fue solicitado su cálculo en forma simbólica y, sin embargo, no pudieron calcularlo cuando se lo enunció en forma de texto contextualizado y en términos de su significado estadístico (ítem «e»), refuerza lo dicho en cuanto al uso de una metodología mecanicista de resolución de ejercicios.

Asimismo, se aprecia que no se comprende demasiado el concepto estadístico de «probabilidad acumulada» dado que un $43 \%$ no asocia su significado ni con el signo matemático ni con expresiones tales como «como mucho», «a lo sumo», «como máximo». 


\section{Conclusión}

Según lo observado, cabe pensar que no se da en igual medida la resolución correcta de un ejercicio de distribución de probabilidad binomial según las consignas estén enunciadas en forma simbólica o de manera coloquial. Resultando mejor la traducción y resolución del ejercicio cuando se va desde lo simbólico a lo textual/coloquial que a la inversa. En tal sentido, podemos concluir que el lenguaje técnico simbólico facilita una resolución más mecanicista que puede prescindir de la comprensión conceptual del objeto enseñado.

En otro aspecto, este estudio evidenció falencias en la comprensión significativa del signo de desigualdad, tanto en su significado asociado a la probabilidad acumulada como al del signo matemático per sé.

\section{Agradecimientos}

El presente trabajo se realizó en el marco del proyecto de investigación PI50120150100053LI «Educación Matemática e Interdisciplinariedad. Eje principal de trabajo: la Modelación», subsidiado por la Universidad Nacional del Litoral. 


\section{Referencias bibliográficas}

- Alisedo, G.; Melgar, S. y Chiocci, C. (1994). Didáctica de las ciencias del lenguaje. Aportes y reflexiones. Paidós.

- D'Amore, B. (2006). Objetos, significados, representaciones semióticas y sentido. En Radford, L. y D'Amore, B. (Eds.). Semiotics, Culture and Matehematical Thinking. Número especial de la revista Relime, 177-196.

- D'Amore, B.; Radford, L. y Bagni, G.T. (2006). Ostacoli epistemologici e prospettiva socioculturale. L'insegnamento della matematica e delle scienze integrate, 29B (1), 11-40.

- Duval, R. (1998). Signe et objet, I et II. Annales de didactique et de sciences cognitives, 6, 139-196.

- Duval, R. (2003). Décrire, visualiser ou raisonner: quels 'apprentissages premiers' de l'activité mathématique? Annales de Didactique et de Sciences Cognitives, 8, 13-62.

- Karmiloff Smith, A. (1994). Más allá de la modularidad. Alianza.

- Radford, L. (2006). Introducción Semiótica y Educación Matemática. Semiotics, Culture and Mathematical Thinking. Número especial de la revista Relime, 7-21. 